\title{
Higher Order Effects in Non Linear Evolution from a Veto in Rapidities
}

\author{
G. Chachamis * a, M. Lublinsky ${ }^{\dagger} \mathbf{b}$, A. Sabio Vera ${ }^{\ddagger} \mathbf{a}$ \\ a II. Institut für Theoretische Physik, Universität Hamburg, \\ Luruper Chaussee 149, 22761 Hamburg, Germany \\ ${ }^{b}$ DESY Theory Group, 22603, Hamburg, Germany
}

December 7, 2018

\begin{abstract}
Higher order corrections to the Balitsky-Kovchegov equation have been estimated by introducing a rapidity veto which forbids subsequent emissions to be very close in rapidity and is known to mimic higher order corrections to the linear BFKL equation. The rapidity veto constraint has been first introduced using analytical arguments obtaining a power growth with energy, $Q_{s}(\mathrm{Y}) \sim e^{\lambda \mathrm{Y}}$, of the saturation scale of $\lambda \sim 0.45$. Then a numerical analysis for the nonlinear Balitsky-Kovchegov equation has been carried out for phenomenological rapidities: when a veto of about two units of rapidity is introduced for a fixed value of the coupling constant of $\alpha_{s}=0.2$ the saturation scale $\lambda$ decreases from $\sim 0.6$ to $\sim 0.3$, and when running coupling effects are taken into account it decreases from $\sim 0.4$ to $\sim 0.3$.
\end{abstract}

*e-mail: chachami@mail.desy.de

$\dagger$ e-mail: lublinm@mail.desy.de

†e-mail: sabio@mail.desy.de 


\section{Introduction}

The high energy behavior of a parton system can be associated to the Balitsky-FadinKuraev-Lipatov (BFKL) dynamics [1. At leading order (LO) the BFKL equation resums contributions of the form $\left(\alpha_{s} \mathrm{Y}\right)^{n}$, with $\mathrm{Y} \sim \ln s$ being a rapidity variable. Linear evolution gives rise to a Pomeron-like behavior of the scattering amplitudes with an intercept $\omega^{\mathrm{BFKL}} \simeq 0.5$. This power growth of the amplitude with energy violates $s$-channel unitarity at rapidities of the order of $\mathrm{Y} \sim 1 / \alpha_{s} \ln 1 / \alpha_{s}$ [2].

A theoretical possibility for the high energy growth of the amplitudes to be modified in a way consistent with unitarity is the idea of parton density saturation [3], which accounts for the possibility of parton annihilation, an essentially nonlinear effect. Present theoretical understanding views a system of saturated partons as a new state of matter called Color Glass Condensate (CGC) (see e.g. Ref. [4] and references therein). Saturation signals have been intensively searched experimentally mostly at HERA and more recently at RHIC. Although there are hints of parton density saturation in the present data, a clean and ultimate signal has not been found so far.

The fundamental quantity characterizing the transition to the saturation regime is the so-called "saturation scale", $Q_{s}(\mathrm{Y})$. The determination of the rate of growth with rapidity of this saturation scale could be of a large importance for, e.g., structure function extrapolations from HERA to LHC kinematics. In the context of the wellknown saturation model of Golec-Biernat and Wusthoff (GBW) [5], the saturation scale grows exponentially as $Q_{s}(\mathrm{Y}) \sim \exp (\lambda \mathrm{Y} / 2)$ with $\lambda \simeq 0.29$.

Within the LO approximation a theoretical tool with solid grounds in perturbative QCD suitable to study saturation phenomena at high energies is the BalitskyKovchegov nonlinear evolution equation $(\mathrm{BKe})^{1}$. This equation reads

$\frac{d N\left(\mathbf{x}_{\mathbf{0 1}}, \mathrm{Y}\right)}{d \mathrm{Y}}=\bar{\alpha}_{s} \int_{\rho} \frac{d^{2} \mathbf{x}_{\mathbf{2}}}{2 \pi} \frac{\mathbf{x}_{\mathbf{0 1}}^{2}}{\mathbf{x}_{\mathbf{0 2}}^{2} \mathbf{x}_{\mathbf{1 2}}^{2}}\left[2 N\left(\mathbf{x}_{\mathbf{0 2}}, \mathrm{Y}\right)-N\left(\mathbf{x}_{\mathbf{0 1}}, \mathrm{Y}\right)-N\left(\mathbf{x}_{\mathbf{0 2}}, \mathrm{Y}\right) N\left(\mathbf{x}_{\mathbf{1 2}}, \mathrm{Y}\right)\right]$

with $\bar{\alpha}_{s} \equiv \alpha_{s} N_{c} / \pi$. In the color dipole approach to high energy scattering the function $N\left(r_{\perp}, \mathrm{Y}, b\right)$ stands for the imaginary part of the amplitude for a dipole of size $r_{\perp}$ elastically scattered at an impact parameter $b$. In this paper the impact parameter dependence of the amplitude will be neglected, considering, in this way, a target of infinite size. $\rho$ is an ultraviolet cutoff needed to regularise the integral which does not appear in physical quantities.

The physical content of Eq. (11) is that of a dipole of size $\mathbf{x}_{10}$ which decays into two other dipoles of size $\mathbf{x}_{\mathbf{1 2}}$ and $\mathbf{x}_{\mathbf{0 2}}$ with a decay probability given by the wave

\footnotetext{
${ }^{1}$ Eq. (11) was originally proposed by Gribov, Levin and Ryskin 3 in momentum space and derived in the double logarithmic approximation by Mueller and Qiu [6. In the leading $\ln 1 / x$ approximation it was obtained by Balitsky using a Wilson Loop Operator Expansion [7. In the form presented in Eq. (1) it was obtained by Kovchegov [8] using the color dipole approach 9] to high energy scattering in QCD. This equation was also obtained by summation of BFKL Pomeron fan diagrams by Braun [10] and, more recently, Bartels, Lipatov and Vacca [11. In the framework of the Color Glass Condensate it was obtained by Iancu, Leonidov and McLerran 12
} 
function $|\Psi|^{2}=\frac{\mathrm{x}_{01}^{2}}{\mathrm{x}_{02}^{2} \mathrm{x}_{12}^{2}}$. These two dipoles can then interact with the target and, with a certain probability, they can do so simultaneously, a possibility accounted for by the non-linear term in Eq. (11). The linear part in Eq. (11) corresponds to the LO BFKL equation describing the evolution with energy of the multiplicity of the fixed size color dipoles. The BKe has been studied both analytically [13, 14, 15, 16] and numerically [10, 17, 18, 19, 20, 21, 22, 23, 24. Phenomenologically the BKe provides a good description of DIS data from HERA [17, 25, 26, 27. It is worth pointing out that the linear part of the BKe is obtained in the leading soft gluon emission approximation keeping the strong coupling fixed and that the large $N_{c}$ limit is used in order to write the nonlinear term as a product of two functions $N$. This limit is at the basis of the color dipole picture and, to a large extent, it corresponds to a mean field theory without dipole correlations. The equation also neglects target correlations, an assumption which might be valid for asymptotically heavy nuclei but not for protons or a realistic nucleus target.

It would be very interesting to go beyond the original BKe and relax some of the underlying assumptions outlined above. At present there is a large activity in this direction. Regarding the contribution of the $N_{c}$ corrections they can be estimated to be up to $15 \%$ [21]. In this publication we would like to focus on the higher order $\alpha_{s}$ corrections which are relevant, in particular, for phenomenological applications.

In principle, unitarity corrections based on LO estimates are expected to be important at rapidities of the order $\mathrm{Y} \sim 1 / \alpha_{s} \ln 1 / \alpha_{s}$, parametrically earlier than the next-to-leading (NLO) corrections which set in at $\mathrm{Y} \sim 1 / \alpha_{s}^{2}$. It is also known that the NLO corrections to the linear BFKL equation significantly decrease the Pomeron intercept thus postponing the arrival of unitarity corrections to higher rapidities.

A complete nonlinear equation at NLO has not been derived yet. In the conventional approach based on $s$-channel unitarity, the forward BFKL kernel is known at NLO [28, 29]. A nonlinear evolution needs the knowledge of the non-forward kernel 30] together with the NLO impact factor [31, 32, 33], both calculations being currently under investigation. However, a NLO study of the triple Pomeron vertex entering the BKe has not been initiated yet. So far, the only exact result which has been reported is due to Balitsky and Belitsky [34] who have been able to compute a single NLO contribution with maximal nonlinearity, the $N^{3}$ term.

There have been some attempts to get insight about saturation at NLO using approximate methods. Triantafyllopoulos [35] has considered the renormalisation group improved NLO BFKL equation with the presence of a saturation boundary. His results show a decrease in the saturation scale growth as a function of rapidity towards the value $\lambda \simeq 0.3$ observed experimentally. A similar type of study based on the NLO BFKL has been recently reported in [36].

In this work we propose a new approach for the study of saturation effects including NLO corrections. We will introduce a constraint in the rapidity of the emitted gluons in the BKe, a so-called "rapidity veto" 37, 38, which, for the linear part of the equation, is known to reproduce the bulk of the NLO corrections. In the next 
section we revise how to introduce a veto in rapidity in the linear BFKL equation and apply this constraint to obtain an estimate of the saturation scale as a function of the veto. In Section 3 we apply the method of rapidity veto to the BKe and study its influence on the energy growth of the saturation scale. In the last Section of this work we present our summary.

\section{The rapidity veto in BFKL and the saturation line}

In the following the introduction of a rapidity veto as in Ref. [37, 38] will be shown. To impose the constraint that subsequent gluon emissions are separated by some minimum interval in rapidity, $\eta$, can be done writing the LO BFKL equation as an integral equation in rapidity, i.e.

$$
f(\mathrm{Y}, \gamma)=\int d \mathrm{Y}^{\prime} \theta\left(\mathrm{Y}-\mathrm{Y}^{\prime}-\eta\right) \bar{\alpha}_{s} \chi(\gamma) f\left(\mathrm{Y}^{\prime}, \gamma\right)
$$

where $\gamma$ corresponds to a Mellin transform in transverse momentum space and $\chi(\gamma)=$ $2 \Psi(1)-\Psi(\gamma)-\Psi(1-\gamma)$ is the eigenvalue of the LO kernel. To go to the representation in the $\omega$ plane we use the transformation

$$
f_{\omega}(\gamma)=\int d \mathrm{Y} e^{-\omega \mathrm{Y}} f(\mathrm{Y}, \gamma)
$$

We can now introduce this in the original equation to obtain

$$
f_{\omega}(\gamma)=\bar{\alpha}_{s} \chi(\gamma) \int d \mathrm{Y}^{\prime} f\left(\mathrm{Y}^{\prime}, \gamma\right) \int_{\mathrm{Y}^{\prime}+\eta} d \mathrm{Y} e^{-\omega \mathrm{Y}}
$$

therefore the effect of imposing the veto on the LO BFKL equation leads to an eigenvalue which is determined by the solution to

$$
\omega=\bar{\alpha}_{s} \chi(\gamma) e^{-\eta \omega}
$$

It is worth noting that the solution to this equation respects the structure of a maximum at $\gamma=\frac{1}{2}+\mathrm{i} \nu$ for $\nu \simeq 0$ so that this region dominates at high energies. This is highlighted in Fig. 1 where the maxima are shown revealing how the original value of the Pomeron intercept decreases from about 0.5 to about 0.3 for a value of the veto of two units of rapidity. This is in agreement with other predictions from studies of the NLO gluon Green's function [39].

Now we calculate the influence of this veto in rapidity on the saturation scale. In the case of forward scattering the amplitude for a dipole of size $1 / Q$ on a dipole of size $1 / Q_{0}$ can be written as

$$
\mathcal{N}\left(Q, Q_{0}, \mathrm{Y}\right)=\int d \gamma \int d \omega \mathcal{N}_{0}(\gamma) \exp (-\gamma L+\omega \mathrm{Y}) \frac{1}{\omega-\bar{\alpha}_{s} \chi(\gamma)}
$$




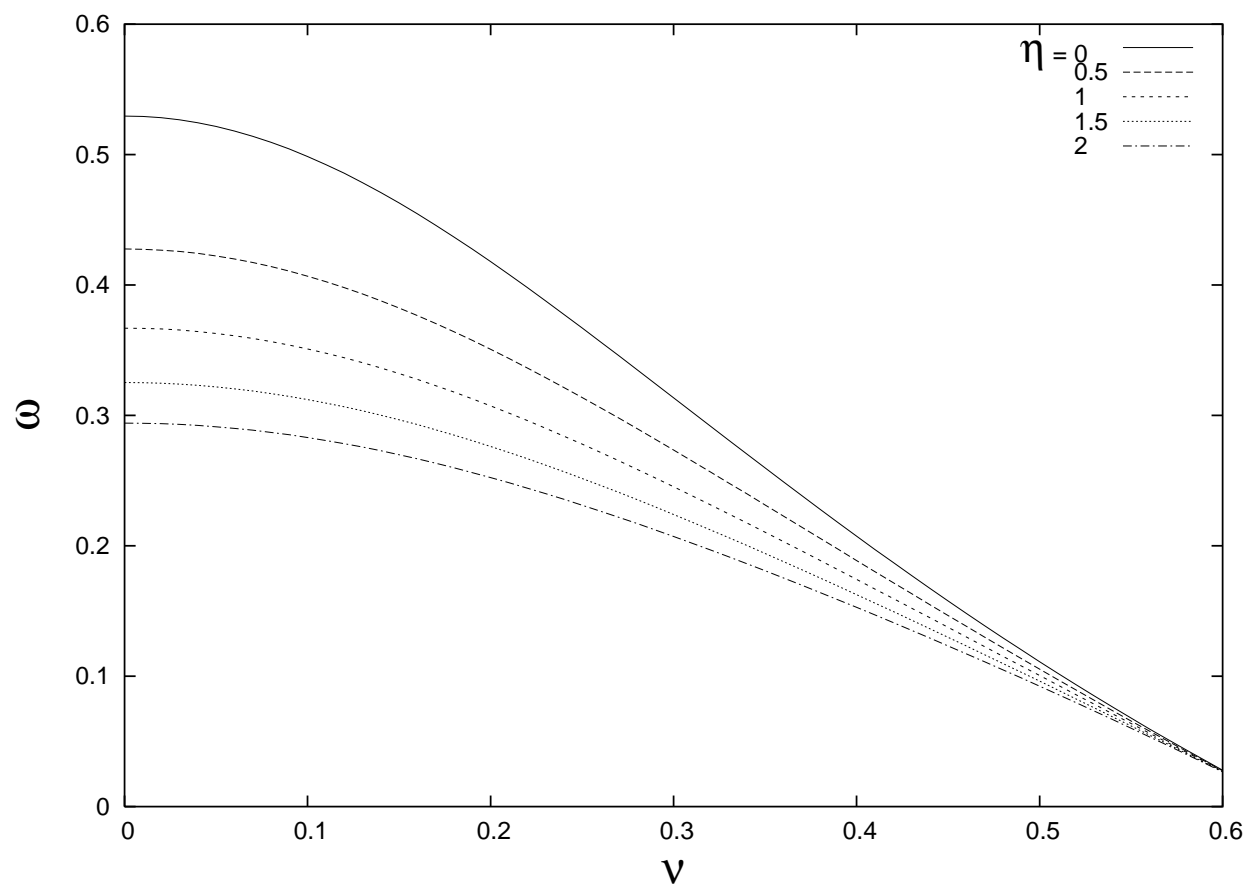

Figure 1: Dependence of the LO eigenvalue of the kernel on the veto upon $\nu$

with $L \equiv \ln Q^{2} / Q_{0}^{2}$. The veto in rapidity is easily introduced via a modified kernel as in Eq. (15):

$$
\mathcal{N}\left(Q, Q_{0}, \mathrm{Y}, \eta\right)=\int d \gamma \int d \omega \mathcal{N}_{0}(\gamma) \exp (-\gamma L+\omega \mathrm{Y}) \frac{1}{\omega-\bar{\alpha}_{s} \chi(\gamma) e^{-\eta \omega}}
$$

The saturation line, $L_{s} \equiv \ln Q_{s}^{2}(\mathrm{Y}) / Q_{0}^{2}$ with $Q_{s}^{2}(\mathrm{Y}=0) \equiv Q_{0}^{2}$, can be defined as that with a stationary exponent:

$$
-\gamma L_{s}+\omega(\gamma, \eta) \mathrm{Y}=0
$$

where the introduction of the veto enforces

$$
\omega(\gamma, \eta)=\bar{\alpha}_{s} \chi(\gamma) \exp (-\eta \omega(\gamma, \eta)) .
$$

At high energies the dominant region is that in the intersection with the saddle point $\bar{\gamma}$

$$
-L_{s}+\left.\frac{\omega(\gamma, \eta)}{d \gamma}\right|_{\gamma=\bar{\gamma}} \mathrm{Y}=0 .
$$

The solution to this system of equations provides an implicit equation for $\bar{\gamma}$ :

$$
\frac{\chi^{\prime}(\bar{\gamma})}{\chi(\bar{\gamma})} \bar{\gamma}-1=\bar{\alpha}_{s} \eta \chi(\bar{\gamma}) \exp \left(1-\frac{\chi^{\prime}(\bar{\gamma})}{\chi(\bar{\gamma})} \bar{\gamma}\right)
$$




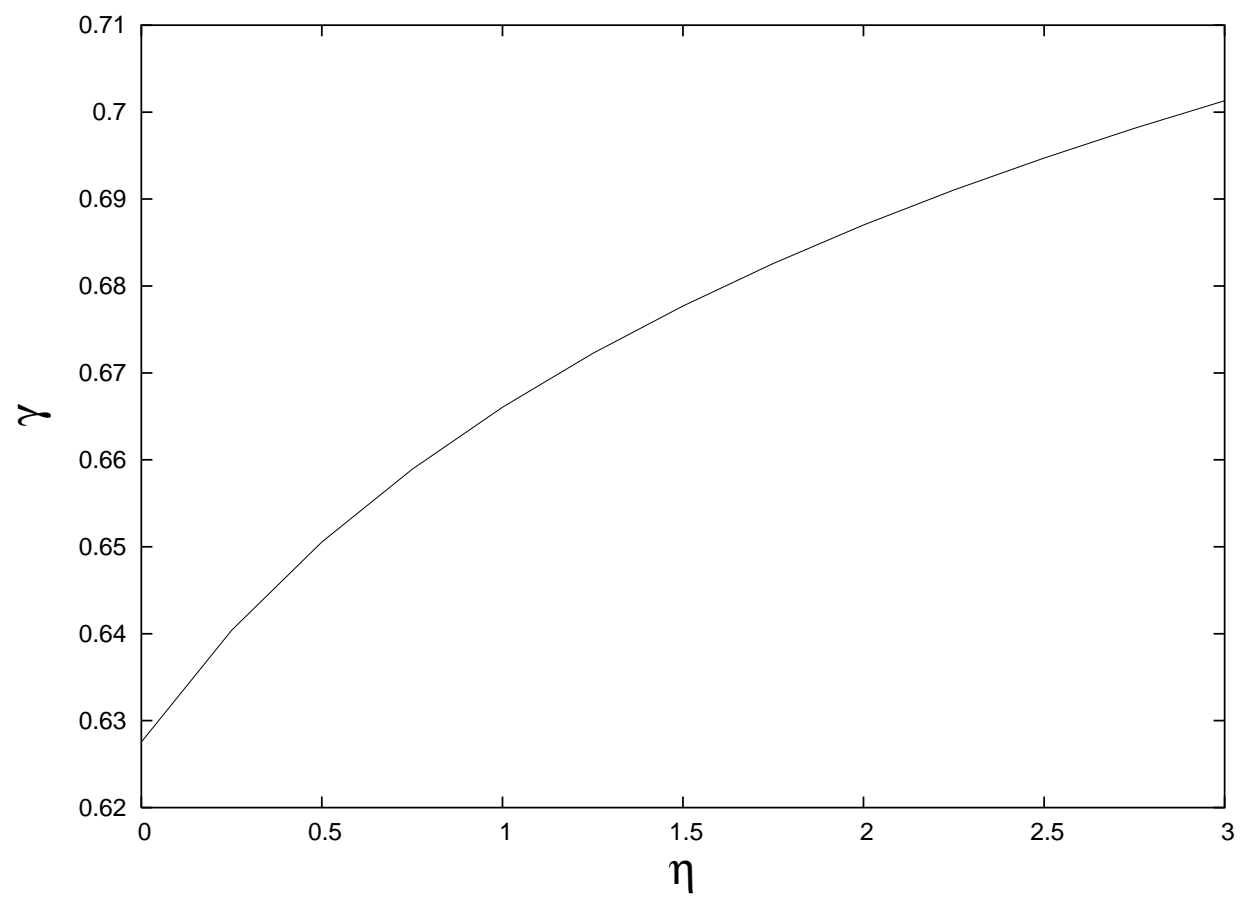

Figure 2: The solution to Eq. (11) as a function of the veto in rapidity, $\eta$.

Consequently, when the rapidity veto is imposed it develops a dependence on the $\bar{\alpha}_{s} \eta$ product, $\bar{\gamma}=\bar{\gamma}\left(\bar{\alpha}_{s} \eta\right)$, and the saturation line reads now

$$
L_{s}=\bar{\alpha}_{s} \frac{\chi(\bar{\gamma})}{\bar{\gamma}} \mathrm{Y} \exp \left(1-\frac{\chi^{\prime}(\bar{\gamma})}{\chi(\bar{\gamma})} \bar{\gamma}\right) \equiv \lambda\left(\bar{\alpha}_{s}, \eta\right) \mathrm{Y} .
$$

For a value of $\alpha_{s}=0.2$ in Fig. 22 we have plotted the dependence of $\bar{\gamma}$ on the rapidity veto $\eta$. The effect of the constraint in rapidity is to increase the value of this critical $\bar{\gamma}$ from the well known $\bar{\gamma} \simeq 0.63$ (a value which is obtained for $\eta \rightarrow 0$ and/or in the limit of $\bar{\alpha}_{s} \rightarrow 0$ ) to about 0.69 for $\eta \sim 2.5$. This is in agreement with the recent results for this quantity of Ref. [36] where a resummed NLL BFKL equation was under study.

The evolution in energy is determined by the $d L_{s} / d \mathrm{Y}=\lambda\left(\bar{\alpha}_{s}, \eta\right)$ derivative. This $\lambda$ term is calculated in Fig. 3 where it can be seen how the effect of the rapidity constraint is to delay the onset of the saturation line by means of decreasing $\lambda$ at larger values of the veto. For zero veto it corresponds to the usual value of this linear coefficient of $\lambda \sim 0.93$, which can be calculated from Eq. (11) setting $\eta=0, \alpha_{s}=0.2$ and then introducing the result for $\bar{\gamma}$ in Eq. (12) reading $\lambda=\bar{\alpha}_{s} \chi(\bar{\gamma}) / \bar{\gamma}$. At a rapidity constraint of $\eta=2.5$ we obtain $\lambda \simeq 0.45$, in agreement with Ref. [36] and larger than that calculated in Ref. 35.

These results have been produced in the case of linear evolution imposing a constraint which reproduces higher order corrections and estimating the position of the saturation line. In the following section we introduce the veto in rapidities directly in the non-linear BKe and study the consequences of this constraint on the evolution for phenomenological rapidities. We will see that the power growth of the saturation 


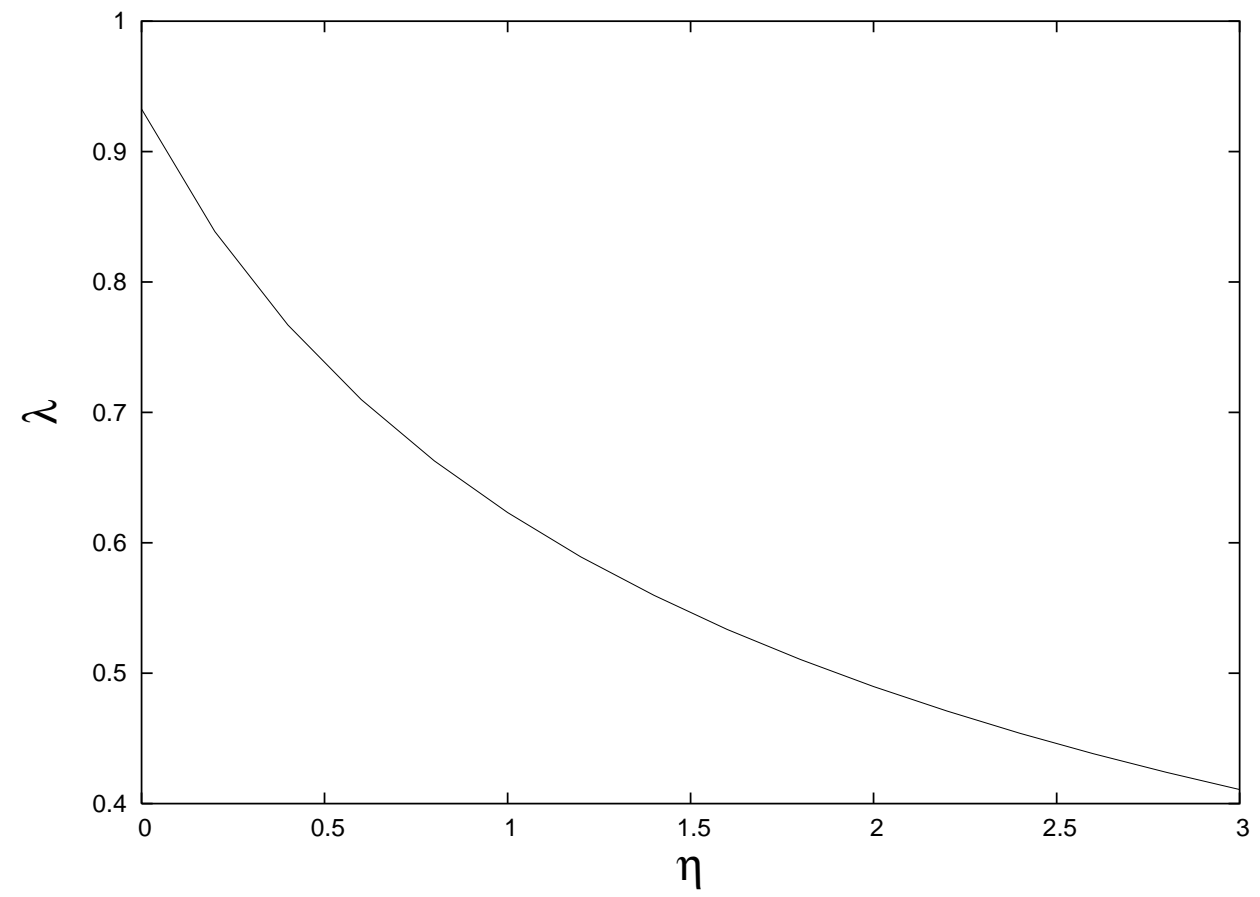

Figure 3: The saturation power (Eq. (12) ) as a function of the veto in rapidity, $\eta$.

scale is slower, even for zero veto, mainly due to preasymptotic effects. We will then show how the effect of the veto in rapidity is very similar to that found in this section: the effective power decreases as the veto is larger.

\section{The rapidity veto in the $\mathrm{BKe}$}

\subsection{The fixed coupling case}

To proceed with the numerical analysis it is convenient to write the BKe for fixed coupling in the integral form

$$
\begin{aligned}
N\left(\mathbf{x}_{\mathbf{0 1}}, \mathrm{Y}\right)= & N\left(\mathbf{x}_{\mathbf{0 1}}, \mathrm{Y}_{0}\right)+\bar{\alpha}_{s} \int_{\mathrm{Y}_{0}}^{\mathrm{Y}} d \mathrm{Y}^{\prime} \int_{\rho} \frac{d^{2} \mathbf{x}_{\mathbf{2}}}{2 \pi} \frac{\mathbf{x}_{\mathbf{0 1}}^{\mathbf{2}}}{\mathbf{x}_{\mathbf{0 2}}^{\mathbf{2}} \mathbf{x}_{\mathbf{1 2}}^{\mathbf{2}}} \times \\
& {\left[2 N\left(\mathbf{x}_{\mathbf{0 2}}, \mathrm{Y}^{\prime}\right)-N\left(\mathbf{x}_{\mathbf{0 1}}, \mathrm{Y}^{\prime}\right)-N\left(\mathbf{x}_{\mathbf{0 2}}, \mathrm{Y}^{\prime}\right) N\left(\mathbf{x}_{\mathbf{1 2}}, \mathrm{Y}^{\prime}\right)\right], }
\end{aligned}
$$

with the initial condition being defined at the rapidity $Y_{0}$. As it was said above the rapidity veto prevents two emissions from being emitted close to each other in rapidity space. In this work we impose this veto in both the linear and non-linear parts of the BKe. In this way we maintain the locality of the recombination process, corresponding to the quadratic term. Hence, the new equation simply reads

$$
\begin{aligned}
N\left(\mathbf{x}_{\mathbf{0 1}}, \mathrm{Y}\right)= & N\left(\mathbf{x}_{\mathbf{0 1}}, \mathrm{Y}_{0}\right)+\bar{\alpha}_{s} \int_{\mathrm{Y}_{0}+\eta}^{\mathrm{Y}-\eta} d \mathrm{Y}^{\prime} \int_{\rho} \frac{d^{2} \mathbf{x}_{\mathbf{2}}}{2 \pi} \frac{\mathbf{x}_{\mathbf{0 1}}^{\mathbf{2}}}{\mathbf{x}_{\mathbf{0 2}}^{\mathbf{2}} \mathbf{x}_{\mathbf{1 2}}^{\mathbf{2}}} \times \\
& {\left[2 N\left(\mathbf{x}_{\mathbf{0 2}}, \mathrm{Y}^{\prime}\right)-N\left(\mathbf{x}_{\mathbf{0 1}}, \mathrm{Y}^{\prime}\right)-N\left(\mathbf{x}_{\mathbf{0 2}}, \mathrm{Y}^{\prime}\right) N\left(\mathbf{x}_{\mathbf{1 2}}, \mathrm{Y}^{\prime}\right)\right] . }
\end{aligned}
$$


In the numerical implementation we work with the differential form of this equation with veto, i.e.,

$$
\begin{aligned}
& \frac{d N\left(\mathbf{x}_{\mathbf{0 1}}, \mathrm{Y}\right)}{d \mathrm{Y}}=\bar{\alpha}_{s} \int_{\rho} \frac{d^{2} \mathbf{x}_{\mathbf{2}}}{2 \pi} \frac{\mathbf{x}_{\mathbf{0 1}}^{\mathbf{2}}}{\mathbf{x}_{\mathbf{0 2}}^{\mathbf{2}} \mathbf{x}_{12}^{\mathbf{2}}} \times \\
& \quad\left[2 N\left(\mathbf{x}_{\mathbf{0 2}}, \mathrm{Y}-\eta\right)-N\left(\mathbf{x}_{\mathbf{0 1}}, \mathrm{Y}-\eta\right)-N\left(\mathbf{x}_{\mathbf{0 2}}, \mathrm{Y}-\eta\right) N\left(\mathbf{x}_{\mathbf{1 2}}, \mathrm{Y}-\eta\right)\right]
\end{aligned}
$$

which highlights the non-locality in rapidity after the constraint has been imposed. Expanding Eq. (15) in $\eta$ (we assume $\eta \ll \mathrm{Y}$ ) one can easily verify that the corrections introduced via veto are proportional to $\alpha_{s}^{2}$ and thus of the NLO.

In the context of the BKe the imposition of the veto has the consequence of a small fluctuation above $N=1$ when the function approaches the unitarity bound. These small fluctuations do not grow with rapidity. Technically this small violation of unitarity has its origin in the fact that the evolution is not stopped at $N(\mathrm{Y})=1$ since the derivative is computed at a retarded rapidity $Y-\eta$, see Eq. (15). This small instability is not surprising since our approach is only an estimate of the NLO corrections. To fully preserve unitarity possibly we would have to introduce a correlation between coordinates and rapidity. Connecting with this point it is worth noticing that a generalization of the BKe proposed in Ref. [40] and amounting to having extra $1-N\left(\mathbf{x}_{\mathbf{0 1}}, Y\right)$ factor in front of the evolution kernel would respect unitarity even in the presence of a rapidity veto. In order to study the behavior of the saturation scale, our analysis will be centered around the transition region $N \simeq 0.5$, which is a region not affected by the above issue.

Eq. (15) will be solved numerically for $x \leq x_{0}=0.01$, which corresponds to rapidities above $\mathrm{Y}_{0} \simeq 4.65$. With this goal in mind we need to specify the initial conditions to the non-linear equation which, in principle, should be fitted to experimental data. Motivated by the phenomenological accuracy of the results in Ref. [17], the same initial conditions as those in that reference are used in the present work. These conditions were fitted to low $x F_{2}$ data for the BKe with no veto and read

$$
N\left(r, \mathrm{Y}_{0}\right)=1-\exp \left(-\alpha_{s} C_{F} r^{2} x G^{\mathrm{CTEQ}} /\left(\pi R^{2}\right)\right)
$$

Here $\alpha_{s}$ is taken to be LO running at the scale $4 / r^{2}$, and $x G^{\mathrm{CTEQ}}$ is the LO CTEQ6 gluon distribution also computed at the scale $4 / r^{2}$. The initial condition in (16) is smoothly extrapolated to $N=1$ at very large distances using the method proposed in Ref. [26] and implemented in Ref. [17. The parameter $R$ stands for the effective proton size, $R^{2}=3.1 \mathrm{GeV}^{-2}$, an output of the fit performed in Ref. [17]. For the numerical implementation of the veto it is necessary to generate the initial conditions in a band of width $\eta$ between $\mathrm{Y}_{0}$ and $\mathrm{Y}_{0}-\eta$. The reason for this becomes clear if we think the evolution in rapidity $\mathrm{Y}$ as a process in which $N(r, \mathrm{Y})$ is the input for the next step which will give us $N(r, \mathrm{Y}+\delta \mathrm{Y})$. In the non zero veto case in order to compute $N(r, \mathrm{Y}+\delta \mathrm{Y})$ one would need to consider $N(r, \mathrm{Y}-\eta+\delta \mathrm{Y})$. Hence, since we start at $\mathrm{Y}_{0}$ we need a band of initial conditions that will span the space between $\mathrm{Y}_{0}-\eta$ and $\mathrm{Y}_{0}$. As there is no evolution in energy along that band we assume the initial conditions to be independent of $\mathrm{Y}$ and equal to $N\left(r, \mathrm{Y}_{0}\right)$ on the band. 
In Fig. 4 the first result for the solution to the BKe is shown. There it can be seen how the amplitude $N$ starts at zero for small transverse distances $r$ (color transparency) to reach the saturation regime $N \sim 1$ at $r$ about $4 \mathrm{GeV}^{-1}$. This trend is general for all values of the rapidity veto but, as a new feature, we observe how the arrival of the saturation of the amplitude is delayed as the veto increases. This first plot was done for a rapidity of 10 and a fixed coupling of $\alpha_{s}=0.2$. In Fig. [5]

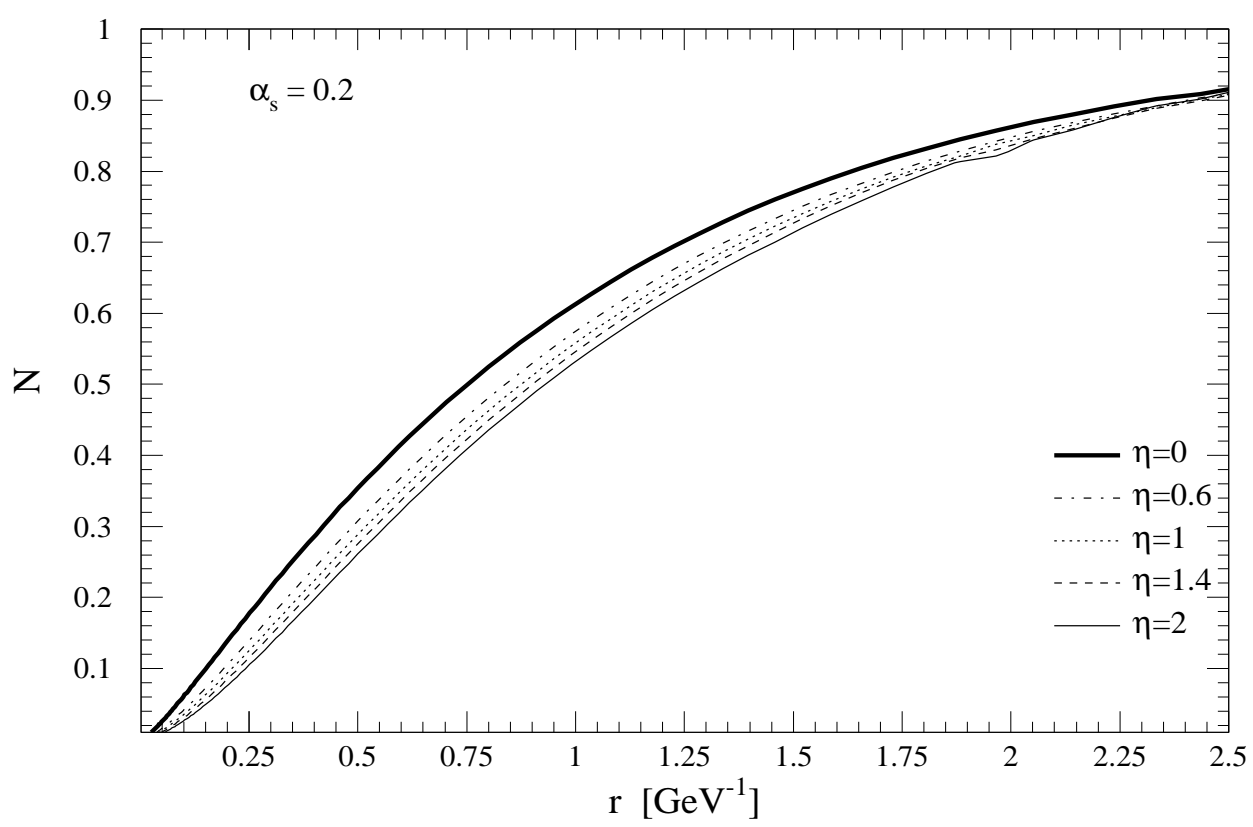

Figure 4: Solution to BKe for different values of the veto as a function of $r$, for $\mathrm{Y}=10$ and $\alpha_{s}=0.2$.

we highlight how in a region of larger rapidity, $\mathrm{Y}=14$ saturation arises earlier in $r$ for the set of initial conditions we have chosen. In this case of larger center-of-mass energy the effect of the veto is more dramatic, considerably delaying the onset of saturation.

The major effect of the rapidity constraint comes when studying how the BK amplitude evolves with energy. As the intercept in the linear part is significantly reduced when higher order corrections are taken into account the saturation of the amplitude comes also later in rapidities. To illustrate this point we plot Fig. 6. where we have chosen a typical value of $r=0.75 \mathrm{GeV}^{-1}$. Once again our estimated NLO corrections do delay the onset of saturation. To make this statement more quantitative we now proceed to study the saturation scale and its dependence with energy. Different definitions of the saturation scale can be associated with the step like function $N$ [26]. They might lead to different normalizations although the energy dependence is qualitatively unique. For the sake of simplicity here we adopt the choice proposed in Ref. [20] where it was taken at a point where $N$ reaches half: 


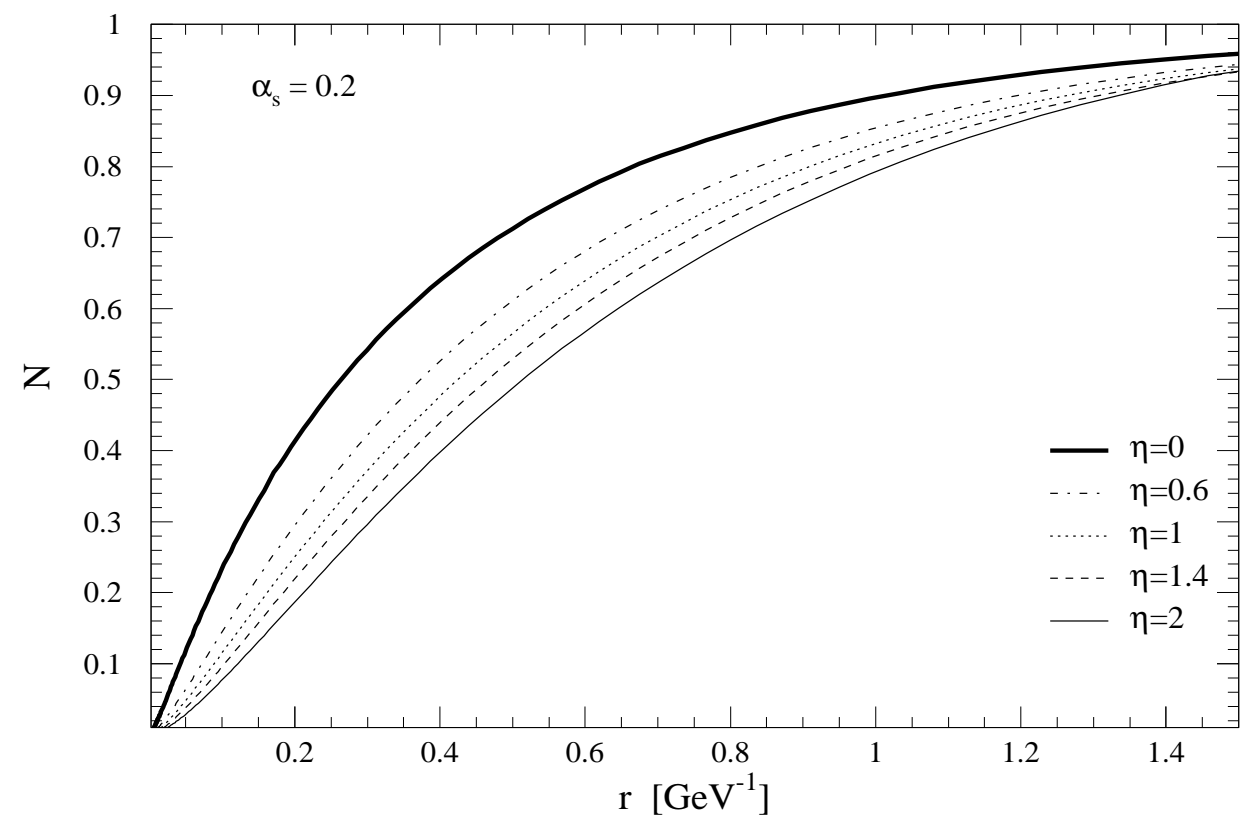

Figure 5: Solution to BKe for different values of the veto as a function of $r$, for $\mathrm{Y}=14$ and $\alpha_{s}=0.2$

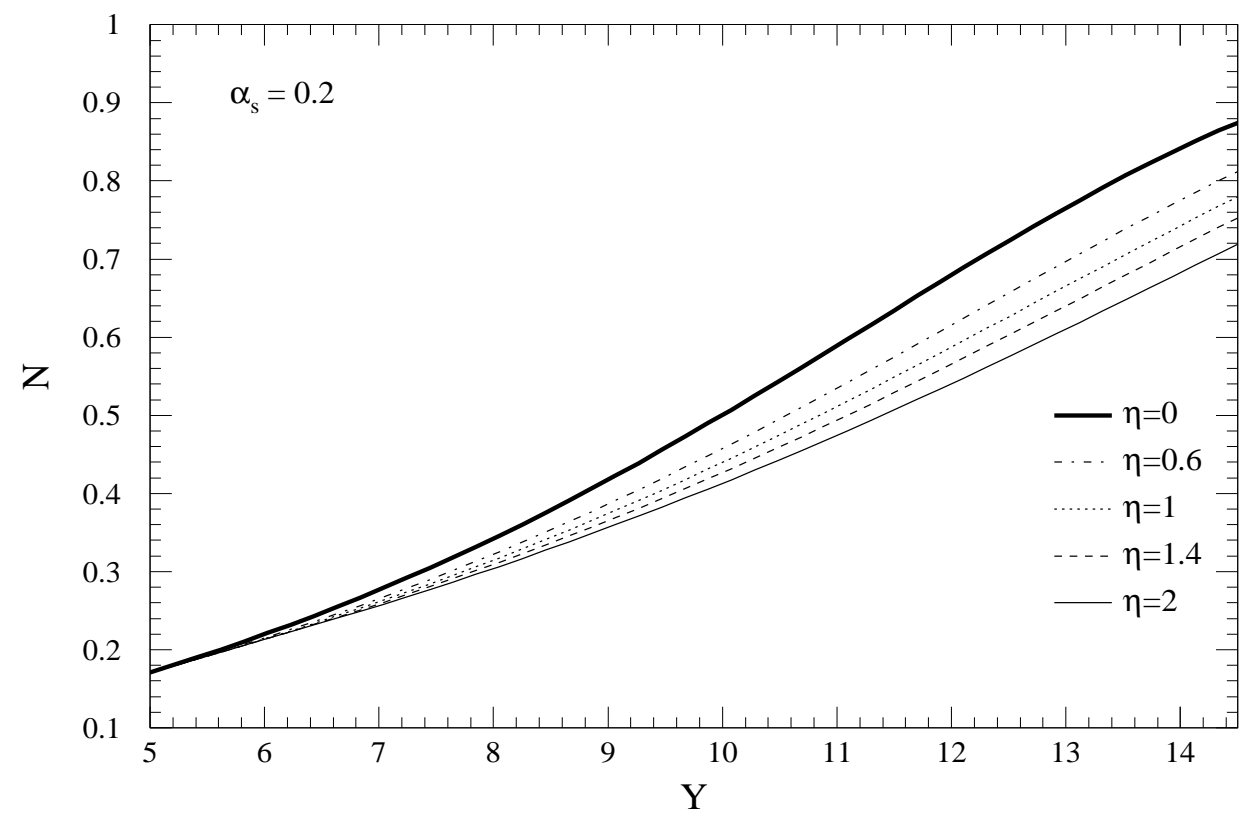

Figure 6: Solution to BKe for different values of the veto as a function of the rapidity $\mathrm{Y}$, for $r=0.75 \mathrm{GeV}^{-1}$ and $\alpha_{s}=0.2$ 


$$
N\left(r_{s}, \mathrm{Y}\right)=\frac{1}{2}
$$

with $r_{s} \equiv 2 / Q_{s}$. For phenomenological applications the behavior of $\ln Q_{s}^{2}$ with rapidity $\mathrm{Y}$ can be fitted linearly as in Eq. (12), i.e.,

$$
Q_{s}^{2}=Q_{0}^{2} e^{\lambda \mathrm{Y}}
$$

The numerical analysis of the rapidity dependence of this saturation scale is carried out in Fig. 7. This plot reflects very clearly how saturation tends to appear later in rapidity, in particular, for the veto which reproduces the NLO intercept, $\eta \sim 2$. We have also performed a linear fit to estimate the linear power of Eq. (12), $\lambda$, this fit was done for phenomenological rapidities between $\mathrm{Y}=10$ and $\mathrm{Y}=15$ so the expected value of the growth cannot be as large as in Fig. 3] due to preasymptotic effects ${ }^{2}$. In fact, $\lambda$ is much smaller at the beginning of the evolution for rapidities up to $Y \simeq 10$. In Fig. 8 we observe the transition power being of the order of 0.65 already at zero

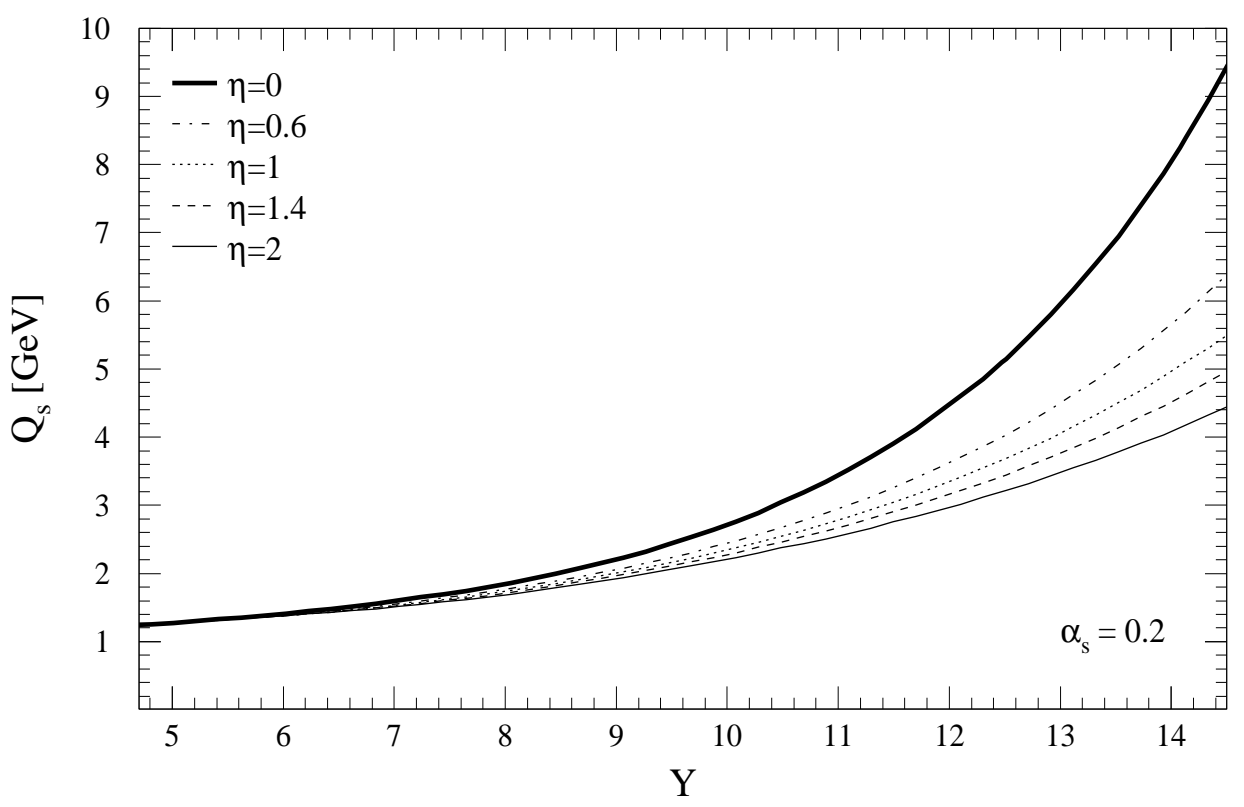

Figure 7: Rapidity dependence of the saturation scale for fixed $\alpha_{s}$ and different vetoes.

$\eta$. This value is smaller than the equivalent obtained in Sec. 2 Remarkably, the dependence on the rapidity veto is of the same functional form as in Fig. 3] with $\lambda$ reaching $\sim 0.31$ at a veto of $\eta=2.5$.

The main conclusion of this section is to confirm the delay in energy space of the arrival of saturation when estimated higher order corrections are introduced in the

\footnotetext{
${ }^{2}$ The preasymptotic effects in saturation scale have been studied in Refs. [41, 15, 16. The numerical size of these terms is large at lower rapidities with the asymptotic values only reached at very large rapidities of the order of $\mathrm{Y} \sim 100$ (see Ref. 24] for a similar discussion).
} 


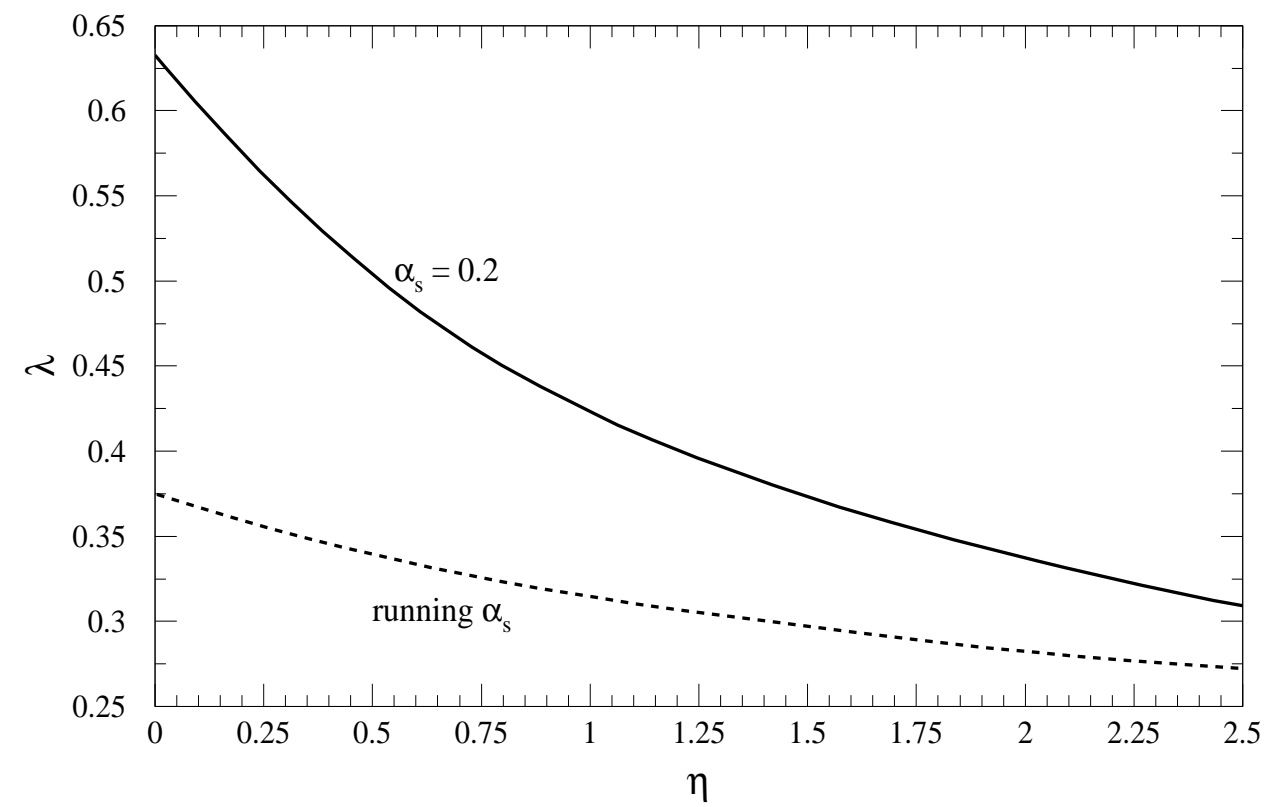

Figure 8: $\eta$ dependence of $\lambda$ with running and fixed $\alpha_{s}=0.2$.

BKe with fixed coupling constant. The numerical results when we introduce the veto on the BKe are consistent with those obtained from a more analytical approach in Sec. 2 based on the BFKL equation with a constraint in rapidity. In the following section the effect of this veto will be studied for phenomenological rapidities and running the QCD coupling.

\subsection{The running coupling case}

The BKe was originally derived for constant $\alpha_{s}$, the introduction of the running is part of the NLO corrections. At present the use of the running $\alpha_{s}$ in the BKe can only be done by modeling. In previous analysis introducing running seems to be phenomenologically favored by the data [17]. This is because the effect of running $\alpha_{s}$ is to bring $\lambda$ down to about 0.3 in the phenomenologically relevant region of $x \geq 10^{-7}$. The main concern of this section is to study the stability of this value of $\lambda \sim 0.3$ when the veto is imposed on top of the running.

Similar to the initial conditions, $\alpha_{s}$ is taken at the leading order running with respect to the external scale $4 / r^{2}$. At large distances $\alpha_{s}$ is frozen at the value $\simeq 0.5$. We have checked that our results are not sensitive to variations of this value.

Let us start with Fig. 9where we again show the region of small $r$ for the amplitude as calculated from the BKe introducing the rapidity constraint, this time running the coupling. As previously found the effect of the higher order corrections is to delay 
saturation. The rapidity chosen for this plot is 14 . It is worth pointing out that the effect of the veto is reduced if we compare Fig. 9 to Fig. 5 , we will go back to this point soon below.

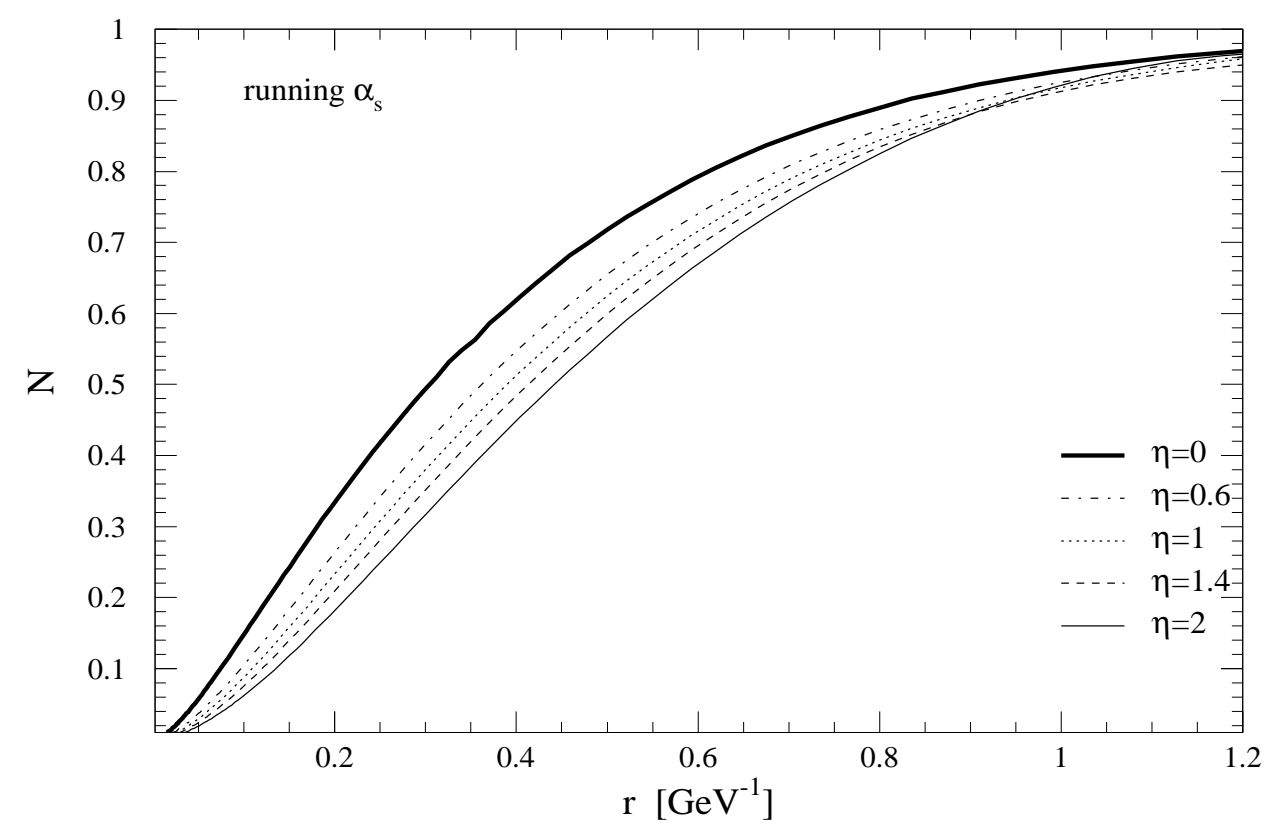

Figure 9: Solution of the BKe for different vetoes as a function of $r$, for $\mathrm{Y}=14$ and running $\alpha_{s}$.

What about the energy dependence of the saturation scale? The answer to this question is plotted in Fig. 10] where the saturation scale is shown as a function of rapidity. The usual delay of the onset of saturation can be again observed although the effect of the estimated higher order corrections is smaller than for the case of fixed coupling in Fig. [7. To make this more explicit we calculate the dependence of the $\lambda$ power in Eq. (12) as a function of the rapidity veto $\eta$ in the case of running coupling ${ }^{3}$ (Fig. (8)) . The main conclusion is that the effect of the veto is not so big in the running coupling case, taking $\lambda$ from $\sim 0.37$ for $\eta=0$ to about 0.27 for $\eta=2.5$. This variation is much smaller than for the fixed coupling case.

The calculations in this section teach us that once the coupling is allowed to run the influence of other higher order corrections is diminished. The prediction for the growth of the saturation scale with energy remains of the order of $\lambda \sim 0.3$ for phenomenological energies independently of the rapidity veto.

As a final remark, we have checked that the solutions to the BKe for both fixed and

\footnotetext{
${ }^{3}$ It is known from analytic studies that, contrary to the fixed coupling case, for running coupling $\ln Q_{s} \sim \sqrt{Y}$. However, again due to large preasymptotic corrections [24, $\ln Q_{s}$ can be fitted linearly in rapidity for a limited range in $\mathrm{Y}$ relevant for phenomenology.
} 


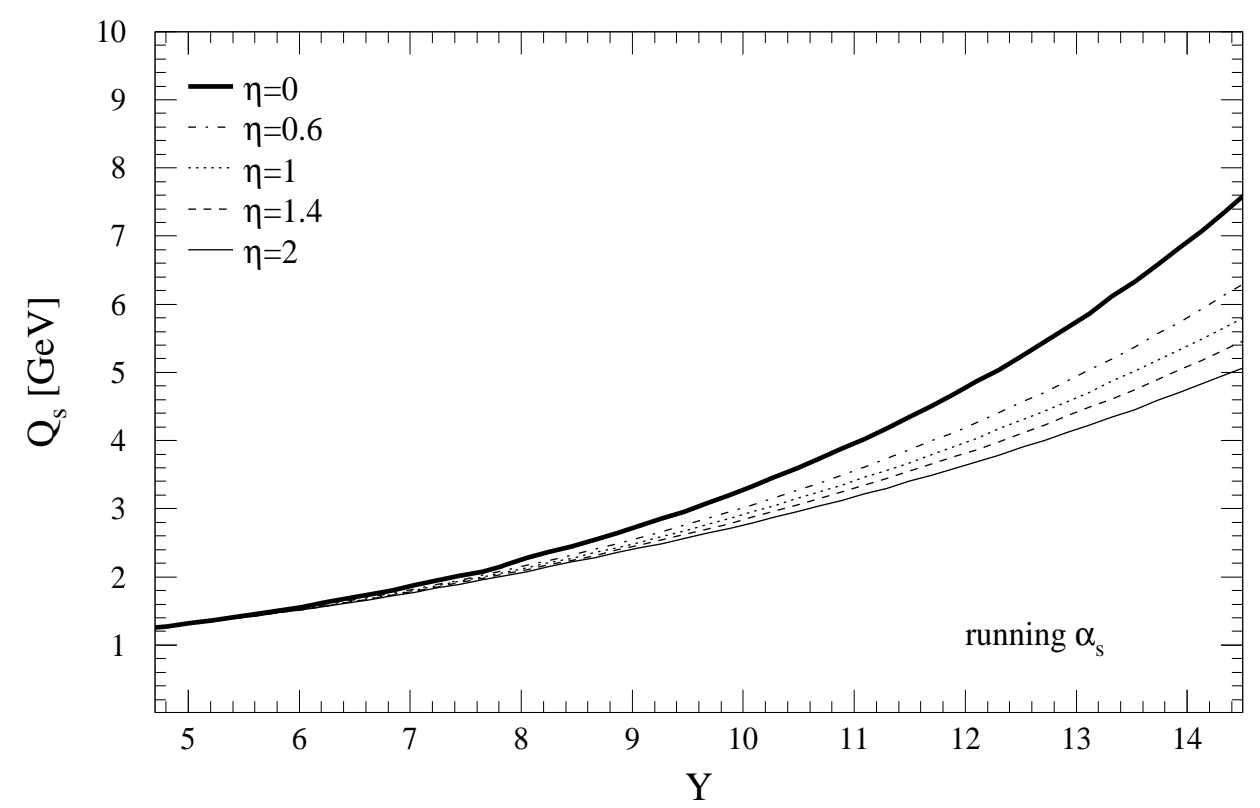

Figure 10: Y dependence of the saturation scale for running $\alpha_{s}$.

running $\alpha_{s}$ cases exhibit the geometrical scaling property [42]. Namely, the amplitude $N(r, \mathrm{Y})$ is a function of the product $\tau=r Q_{s}(\mathrm{Y})$, that is $N(\tau)$. Scaling holds in the saturation domain $\tau>2$ and extends to a much broader region $\tau<<2$. For the BKe with $\eta=0$ scaling was shown in Refs. [18, 26, 19]. We have found that the scaling is still present after introducing a non-zero veto, suggesting the full NLO amplitude to be also a scaling function.

\section{Conclusions}

In this work higher order corrections to the Balitsky-Kovchegov equation have been estimated. This estimate has been based on the introduction of a so-called "rapidity veto", which forbids two emissions to be very close in rapidity. It is known from Ref. [37, 38] that the introduction of a veto, $\eta$, of $\sim 2.5$ units of rapidity mimics the Pomeron intercept predicted by other resummations of the NLO BFKL equation. We have estimated these higher order corrections first using analytical arguments imposing the rapidity veto on the LO BFKL equation obtaining a power growth of the saturation scale of $\lambda \simeq 0.45$, for $\alpha_{s}=0.2$, consistent with that calculated in Ref. 36 and larger than that obtained in Ref. 35, our main result being Eq. (11), Eq. (12) and shown in Fig. 3.

We have then pursued a numerical analysis of the introduction of the rapidity constraint in the full Balitsky-Kovchegov equation for phenomenological rapidities, 
without using asymptotic arguments. For a fixed coupling constant of 0.2 we observe that the power $\lambda$ decreases from $\sim 0.65$ for zero veto to $\sim 0.31$ for $\eta=2.5$, with a dependence on the veto very similar to the previous analytical study, the main result plotted in Fig. 8. When running coupling effects are also taken into account the effect of imposing the veto is not so important taking $\lambda$ at no veto from $\sim 0.37$ to $\sim 0.27$ for $\eta=2.5$ (Fig. 8) .

As follows from the present analysis the running coupling effects account for the bulk of the NLO corrections to the BKe (see Fig. 8). Given that, as shown in [24], the dipole amplitude is not sensitive to the way the running is introduced, this suggests that phenomenological analysis including running coupling effects, as in [17], do provide reliable predictions.

\section{Acknowledgements:}

We would like to thank Nestor Armesto, Jochen Bartels, Jeff Forshaw, Krzysztof Kutak, Eugene Levin, Lev Lipatov, Misha Ryskin, Anna Stasto and James Stirling for fruitful discussions and interest in this work. A.S.V. would like to thank the CERN Theory Division for hospitality, his contribution to this work was supported by an Alexander von Humboldt Postdoctoral Fellowship. G.C. is supported by the Graduiertenkolleg "Zukünftige Entwicklungen in der Teilchenphysik".

\section{References}

[1] L. N. Lipatov, Sov. J. Nucl. Phys. 23 (1976) 338, E. A. Kuraev, L. N. Lipatov, V. S. Fadin, Sov. Phys. JETP 45 (1977) 199, I. I. Balitsky, L. N. Lipatov, Sov. J. Nucl. Phys. 28 (1978) 822.

[2] A. H. Mueller, Phys. Lett. B 396 (1997) 251.

[3] L. V. Gribov, E. M. Levin, and M. G. Ryskin, Nucl. Phys. B 188 (1981) 555; Phys. Rep. 100 (1983) 1.

[4] L. D. McLerran, Lect. Notes Phys. 583 (2002) 291; hep-ph/0104285

[5] K. Golec-Biernat and M. Wusthoff, Phys. Rev. D 59 (1999) 014017.

[6] A. H. Mueller and J. Qiu, Nucl. Phys. B 268 (1986) 427.

[7] Ia. Balitsky, Nucl.Phys. B 463 (1996) 99.

[8] Yu. Kovchegov, Phys. Rev. D 60 (2000) 034008.

[9] A. H. Mueller, Nucl. Phys. B 415 (1994) 373.

[10] M. Braun, Eur. Phys. J. C 16 (2000) 337.

[11] J. Bartels, L.N. Lipatov, and J.P. Vacca, hep-ph/0404110. 
[12] E. Iancu, A. Leonidov, and L. McLerran, Nucl. Phys. A 692 (2001) 583.

[13] Yu. Kovchegov, Phys. Rev. D 61 (2000) 074018; E. Levin and K. Tuchin, Nucl. Phys. B 573 (2000) 833; Nucl. Phys. A 691 (2001) 779.

[14] A. Kovner and U.A. Wiedemann, Phys. Rev. D 66 (2002) 051502.

[15] E. Iancu, K. Itakura and L. McLerran, Nucl. Phys. A 708 (2002) 327.

[16] S. Munier and R. Peschanski, Phys. Rev. D 69 (2004) 034008; Phys. Rev. Lett. 91 (2003) 232001; hep-ph/0401215.

[17] E. Gotsman, E. Levin, M. Lublinsky, and U. Maor, Eur. Phys. J C 27 (2003) 411; parameterizations are available at www.desy.de/ lublinm/.

[18] N. Armesto and M. Braun, Eur. Phys. J. C 20 (2001) 517.

[19] K. Golec-Biernat, L. Motyka, A. Stasto, Phys. Rev. D 65 (2002) 074037.

[20] M. Lublinsky, E. Gotsman, E. Levin, and U. Maor, Nucl. Phys. A 696 (2001) 851.

[21] K. Rummukainen and H. Weigert, Nucl. Phys. A 739 (2004) 183.

[22] K. Golec-Biernat and A.M. Stasto, Nucl. Phys. B 668 (2003) 345;

E. Gotsman, M. Kozlov, E. Levin, U. Maor, and E. Naftali, hep-ph/0401021.

[23] K. Kutak and A. Stasto, hep-ph/0408117.

[24] J. L. Albacete, N. Armesto, J. G. Milhano, C. A. Salgado and U. A. Wiedemann, hep-ph/0408216.

[25] E. Gotsman, E. Levin, M. Lublinsky, U. Maor, and E. Naftali, Acta Phys. Polon. B 34 (2003) 3255;

J. Bartels, E. Gotsman, E. Levin, M. Lublinsky, and U. Maor, Phys. Rev. D 68 (2003) 054008; Phys. Lett. B 556 (2003) 114;

E. Levin and M. Lublinsky, Nucl. Phys. A 696 (2001) 833; Phys. Lett. B 521 (2001) 233.

[26] M. Lublinsky, Eur. Phys. J. C 21 (2001) 513.

[27] E. Iancu, K. Itakura and S. Munier Phys.Lett. B 590 (2004) 199.

[28] V. S. Fadin, L. N. Lipatov, Phys. Lett. B 429 (1998) 127.

[29] M. Ciafaloni, G. Camici, Phys. Lett. B 430 (1998) 349.

[30] V. S. Fadin, Nucl. Phys. Proc. Suppl. 99A (2001) 204.

[31] V. S. Fadin, D. Y. Ivanov and M. I. Kotsky, Nucl. Phys. B 658 (2003) 156. 
[32] V. S. Fadin, R. Fiore, M. I. Kotsky and A. Papa, Phys. Rev. D 61 (2000) 094005.

[33] J. Bartels and A. Kyrieleis, hep-ph/0407051,

J. Bartels, D. Colferai, S. Gieseke and A. Kyrieleis, Phys. Rev. D 66 (2002) 094017,

J. Bartels, S. Gieseke and A. Kyrieleis, Phys. Rev. D 65 (2002) 014006,

J. Bartels, S. Gieseke and C. F. Qiao, Phys. Rev. D 63 (2001) 056014 [Erratumibid. D 65 (2002) 079902].

[34] I. Balitsky and A. Belitsky, Nucl. Phys. B 629 (2002) 290.

[35] D.N. Triantafyllopoulos, Nucl. Phys. B 648 (2003) 293.

[36] V. A. Khoze, A. D. Martin, M. G. Ryskin and W. J. Stirling, hep-ph/0406135.

[37] C. R. Schmidt, Phys. Rev. D 60 (1999) 074003.

[38] J. R. Forshaw, D. A. Ross and A. Sabio Vera, Phys. Lett. B 455 (1999) 273.

[39] G.P. Salam, JHEP8907 (1998) 19,

M. Ciafaloni and D. Colferai, Phys. Lett.B452 (1999) 372,

M. Ciafaloni, D. Colferai and G.P. Salam, Phys. Rev. D60 (1999) 114036,

R.S. Thorne, Phys. Rev. D60 (1999) 054031,

J. R. Forshaw, D. A. Ross and A. Sabio Vera, Phys. Lett. B 498 (2001) 149,

G. Altarelli, R. D. Ball and S. Forte, Nucl. Phys. B 575 (2000) 313, Nucl. Phys. B 621 (2002) 359, Nucl. Phys. B 674 (2003) 459,

M. Ciafaloni, D. Colferai, G. P. Salam and A. M. Stasto, Phys. Lett. B 576 (2003) 143, Phys. Rev. D 68 (2003) 114003, Phys. Lett. B 587 (2004) 87,

J. R. Andersen and A. Sabio Vera, Phys. Lett. B 567 (2003) 116, Nucl. Phys. B 679 (2004) 345, hep-th/0406009.

[40] E. Levin and M. Lublinsky, Nucl. Phys. A 730 (2004) 191.

[41] A.H. Mueller and D.N. Triantafyllopoulos, Nucl. Phys. B 640 (2002) 331.

[42] A. M. Stasto, K. Golec-Biernat and J. Kwiecinski, Phys. Rev. Lett. 86 (2001) 596. 